Check for updates

Cite this: Nanoscale Adv., 2019, 1, 3506

\section{Instantaneous, room-temperature, open-air atmosphere, solution-phase synthesis of perovskite quantum dots through halide exchange employing non-metal based inexpensive $\mathrm{HCl} / \mathrm{HI}$ : ensemble and single particle spectroscopy $\dagger$}

Saptarshi Mandal, (DD ${ }^{a}$ Debjit Roy, ${ }^{a}$ Chayan K. De, ${ }^{a}$ Swarnali Ghosh, ${ }^{a}$ Mrinal Mandal, (D) ${ }^{a}$ Ananya Das ${ }^{a}$ and Prasun K. Mandal (D)*ab

\begin{abstract}
Herein, the instantaneous synthesis of highly crystalline, uniform-sized (ca. $11.3 \pm 0.1 \mathrm{~nm}$ ), blue-to-green to yellow to red-emitting all-inorganic perovskite quantum dots $\left(\mathrm{CsPbBr}_{3}\right.$ and mixed halide PQDs) was achieved at room temperature under an open-air atmosphere (no glove box) through halide exchange in the solution phase employing easily available, inexpensive non-metal-based halide sources such as $\mathrm{HCl}$ and $\mathrm{HI}$. No complicated pre-treatment of the halide source was required. Moreover, these PQDs were stable for a few weeks under an open-air atmosphere. The PL emission spectra are quite narrow, and the PLQYs are quite high ( $80 \%$ for even $\mathrm{Br} / \mathrm{l}$ mixed PQDs). At the single particle level, the $\langle\mathrm{ON}$ fraction〉 has been noted to vary from $75 \%$ to $85 \%$ for different PQDs, the $m_{O N}$ values are close to 1.0 , and the $m_{\mathrm{OFF}}$ values are $>1.5$. The latter indicates that long $\mathrm{ON}$ durations are more probable. The increase in the ON event truncation time (from 2.7 to $4.0 \mathrm{~s}$ ) and the concomitant decrease in the OFF event truncation time (from 6.6 to $4.3 \mathrm{~s}$ ) could be correlated with the increase in the PLQY (from 0.55 to 0.75 ). In addition, an interesting memory effect could be observed in both the ON and the OFF event durations.
\end{abstract}

Received 28th June 2019 Accepted 6th July 2019

DOI: 10.1039/c9na00406h

rsc.li/nanoscale-advances and $\mathrm{PbX}_{2}, \mathrm{X}=\mathrm{Cl}, \mathrm{Br}$, and $\left.\mathrm{I}\right)^{18-21}$ and/or poor solubility of metal salts ( $\mathrm{MX}_{2} / \mathrm{MX}, \mathrm{M}=\mathrm{Zn}, \mathrm{Mg}, \mathrm{Cu}, \mathrm{Ca}$, and $\mathrm{K} ; \mathrm{X}=\mathrm{Cl}, \mathrm{Br}$, and $\left.\mathrm{I}\right)$ in toluene/DMF at RT are the matters of concern. ${ }^{22-26}$ The mixing of the previously synthesized $\mathrm{CsPbX}_{3}(\mathrm{X}=\mathrm{Cl}, \mathrm{Br}$, and I) (through the hot-injection technique) at room temperature (RT) is another way to solve this problem. ${ }^{27}$ However, the precursors of PQDs $\left(\mathrm{CsPbX}_{3}, \mathrm{X}=\mathrm{Cl} / \mathrm{Br} / \mathrm{I}\right)$ are constructed using the hotinjection technique under an inert atmosphere; this is again a matter of concern.

Therefore, it would be interesting if all these reactions could be performed at RT under an open atmosphere, the halide exchange reactions could be performed using easily available and inexpensive non-metal-based halides such as $\mathrm{HCl} / \mathrm{HI}$, and all the reactions could be instantaneous in nature. To the best of our knowledge, there is no study in the literature in which both the synthesis of $\mathrm{CsPbBr}_{3}$ as well as the halide exchange reactions have been performed in the solution phase at RT under an open-air atmosphere using non-metal-based, easily available and inexpensive halide sources such as $\mathrm{HCl} / \mathrm{HI}$. Therefore, it would be highly advantageous if all the abovementioned propositions could be achieved; however, clearly, the achievement of these propositions is very challenging; thus, in this study, we report the instantaneous synthesis (i.e., the overall synthesis takes less time) of both $\mathrm{CsPbBr}_{3}$ and halide-exchanged PQDs at $\mathrm{RT}$ (i.e., without heating) under an open-air atmosphere (i.e., 
without using any glove box). Importantly, the halide exchange reaction was carried out in the liquid phase using inexpensive, easily available, non-metal-based halide sources such as $\mathrm{HCl}$ and HI. Moreover, no complicated pre-treatment of the halide precursors, as reported in the literature, was necessary for our successful solution-phase halide exchange reaction as we could directly use $\mathrm{HCl}$ and $\mathrm{HI}$. In short, we could achieve (Scheme 1) all the abovementioned challenging propositions. To the best of our knowledge, to date, no such complete study has been reported in the literature.

\section{Results and discussion}

At first, $\mathrm{CsPbBr}_{3}$ was synthesized adopting the roomtemperature supersaturated recrystallization method with a modified procedure. ${ }^{\mathbf{2 4} 28}$ In a typical synthesis, $\mathrm{CsPbBr}_{3}$ was instantaneously formed at RT under an open-air atmosphere (Video S1 $\dagger$ ). Moreover, mixed halide PQDs were synthesized in the solution phase instantaneously at room temperature under an open-air atmosphere (henceforth, termed as IRTOA) using the non-metal based, easily available, and inexpensive $\mathrm{HCl} / \mathrm{HI}$ as the halide exchange material. The instantaneous synthesis of both $\mathrm{CsPb}(\mathrm{Br} / \mathrm{Cl})_{3}$ and $\mathrm{CsPb}(\mathrm{Br} / \mathrm{I})_{3}$ mixed halide PQDs could be visualized (Videos $\mathrm{S} 1 \uparrow$ and $\mathrm{S} 2$ ); the instantaneous nature of the halide exchange reaction could be controlled by choosing a proper solvent pair (see $\mathrm{S} 1 \dagger$ ).

After the formation of the mixed $\mathrm{CsPb}(\mathrm{Br} / \mathrm{Cl})_{3}$ PQD $\left(\lambda_{\mathrm{em}}=\right.$ $430 \mathrm{~nm})$, Cl-exchanged mixed $\mathrm{CsPb}(\mathrm{Br} / \mathrm{Cl})_{3}$ PQDs with increasing emission maxima were formed through the IRTOA method with the addition of $\mathrm{HBr}$. Similarly, after the formation of the mixed $\operatorname{CsPb}(\mathrm{Br} / \mathrm{I})_{3} \mathrm{PQD}\left(\lambda_{\mathrm{em}}=630 \mathrm{~nm}\right)$, the I-exchanged mixed $\mathrm{CsPb}(\mathrm{Br} / \mathrm{I})_{3}$ PQDs were formed following the IRTOA process with the addition of $\mathrm{HBr}$ (Fig. S3†). Thus, the reversibility of this halide exchange process through the IRTOA method could be evidenced. Herein, note that in our case, even

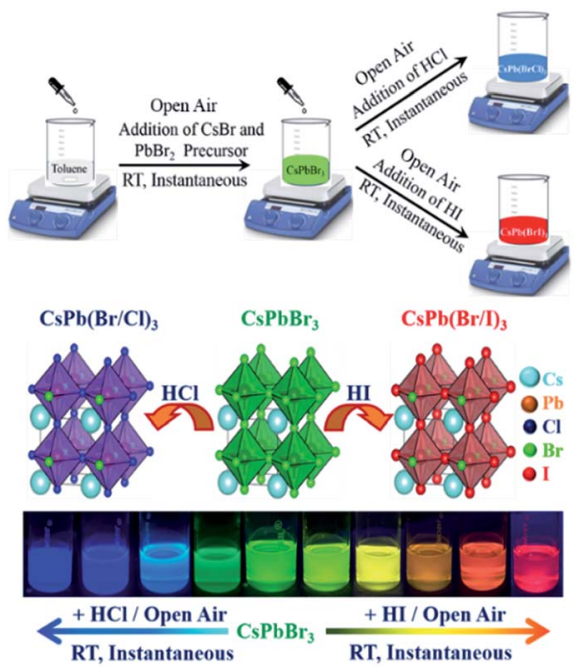

Scheme 1 Room-temperature, open-air atmosphere, instantaneous synthesis of $\mathrm{CsPbBr}_{3}$ and mixed halide perovskites using non-metal and inexpensive halide sources such as $\mathrm{HCl} / \mathrm{HI}$. the $\mathrm{CsPbBr}_{3}$ PQD was synthesized through the IRTOA method. Thus, all steps of the reactions were instantaneous and carried out at RT under an open-air atmosphere. The instantaneous reaction saves a lot of time, the RT synthesis saves a lot of heat/ electrical energy, the open-atmosphere synthesis reduces the difficulties experienced during an inert-atmosphere synthesis procedure, and the usage of easily available and inexpensive $\mathrm{HCl} / \mathrm{HBr} / \mathrm{HI}$ as the halide exchange source materials reduces the cost of the reaction. All these parameters will be extremely useful for the large-scale synthesis of the all-inorganic blue to green to red-emitting PQDs necessary for various applications.

Regarding stability under an ambient air condition, the PQD 1-5 (the mixed halide $\mathrm{CsPb}(\mathrm{Br} / \mathrm{Cl})_{3}$ and $\mathrm{CsPbBr}_{3}$ PQDs) were stable in the solution (toluene) phase for a month (Fig. $\mathrm{S} 4(\mathrm{a}) \dagger$ ). However, the PQD 6-10 (the mixed halide $\mathrm{CsPb}(\mathrm{Br} / \mathrm{I})_{3}$ PQDs) were not very stable in a toluene medium, and the intensity dropped down to $50 \%$ in less than two weeks (Fig. S4(a) $\dagger$ ). The stability of these PQDs was slightly better in $n$-octane as compared to that in the toluene medium (Fig. S4(b) †). Thus, the blue to red-emitting PQDs synthesized through the IRTOA process are as stable (if not more stable) as the PQDs synthesized through the hot injection, inert-atmosphere synthesis using expensive halide exchange materials.

Herein, since all the PQDs were synthesized via a completely different IRTOA procedure in the solution phase using inexpensive $\mathrm{HX}(\mathrm{X}=\mathrm{Cl} / \mathrm{I})$ as compared to those synthesized using the methods (requiring several hours, high temperatures, an inert atmosphere inside glove box, and the use of expensive metal halide sources) reported in the literature, it was necessary to determine the quality of the PQDs. The successful halide exchange reaction and gradual change in halide concentration could be confirmed using the EDAX peak positions (Fig. S5 $\dagger$ ), HRTEM and PXRD (Fig. 1). As can be observed from Fig. 1(a), the sizes of the mixed halide PQDs were not significantly altered because of the halide exchange reaction. The average size of the Cl-exchanged $\mathrm{CsPb}(\mathrm{Br} / \mathrm{Cl})_{3} \mathrm{PQD}$ was $11.31 \pm 0.96 \mathrm{~nm}\left(\lambda_{\mathrm{em}}=430\right.$ $\mathrm{nm})$, that of $\mathrm{CsPbBr}_{3}$ was $11.26 \pm 0.84 \mathrm{~nm}\left(\lambda_{\mathrm{em}}=513 \mathrm{~nm}\right)$ and that of the I-exchanged $\mathrm{CsPb}(\mathrm{Br} / \mathrm{I})_{3} \mathrm{PQD}$ was $11.39 \pm 0.80 \mathrm{~nm}$ $\left(\lambda_{\text {em }}=630 \mathrm{~nm}\right)$ (Fig. 1(a)). The excellent HRTEM images (Fig. 1(a)) as well as the bright spots in the FFT pattern (Fig. 1(a)) confirm the very high crystallinity of the PQDs synthesized using the abovementioned ingenious approach. The constancy of the quality and the size/shape of the halide-exchanged PQDS as compared to those of $\mathrm{CsPbBr}_{3}$ confirmed that no strong detrimental effect was caused by the $\mathrm{H}^{+}$ion of $\mathrm{HX}(\mathrm{X}=\mathrm{Cl} / \mathrm{I})$. The inter-planar spacings obtained from the HRTEM images have been calculated to be $0.57 \mathrm{~nm}$ for the Cl-exchanged $\mathrm{CsPb}(\mathrm{Br} / \mathrm{Cl})_{3}$ PQD $\left(\lambda_{\text {em }}=430 \mathrm{~nm}\right), 0.58 \mathrm{~nm}$ for the $\mathrm{CsPbBr}_{3} \mathrm{PQD}\left(\lambda_{\mathrm{em}}=513\right.$ $\mathrm{nm})$ and $0.61 \mathrm{~nm}$ for the I-exchanged $\operatorname{CsPb}(\mathrm{Br} / \mathrm{I})_{3}$ PQD $\left(\lambda_{\mathrm{em}}=\right.$ $630 \mathrm{~nm})$. These inter-planar spacings correspond to the $\{100\}$ plane. ${ }^{27}$ The inter-planar spacings corresponding to the $\{100\}$ plane have also been calculated from the PXRD patterns (Fig. 1(b)). The inter-planar spacing obtained via HRTEM and PXRD matches quite well with that of the $\{100\}$ plane; thus, because of the exchange of the smaller sized $\mathrm{Cl}^{-}(1.81 \AA)$ in place of the larger sized $\mathrm{Br}^{-}(1.96 \AA)$, the inter-planar spacing decreases from $0.58 \mathrm{~nm}$ to $0.57 \mathrm{~nm}$. Similarly, because of the 

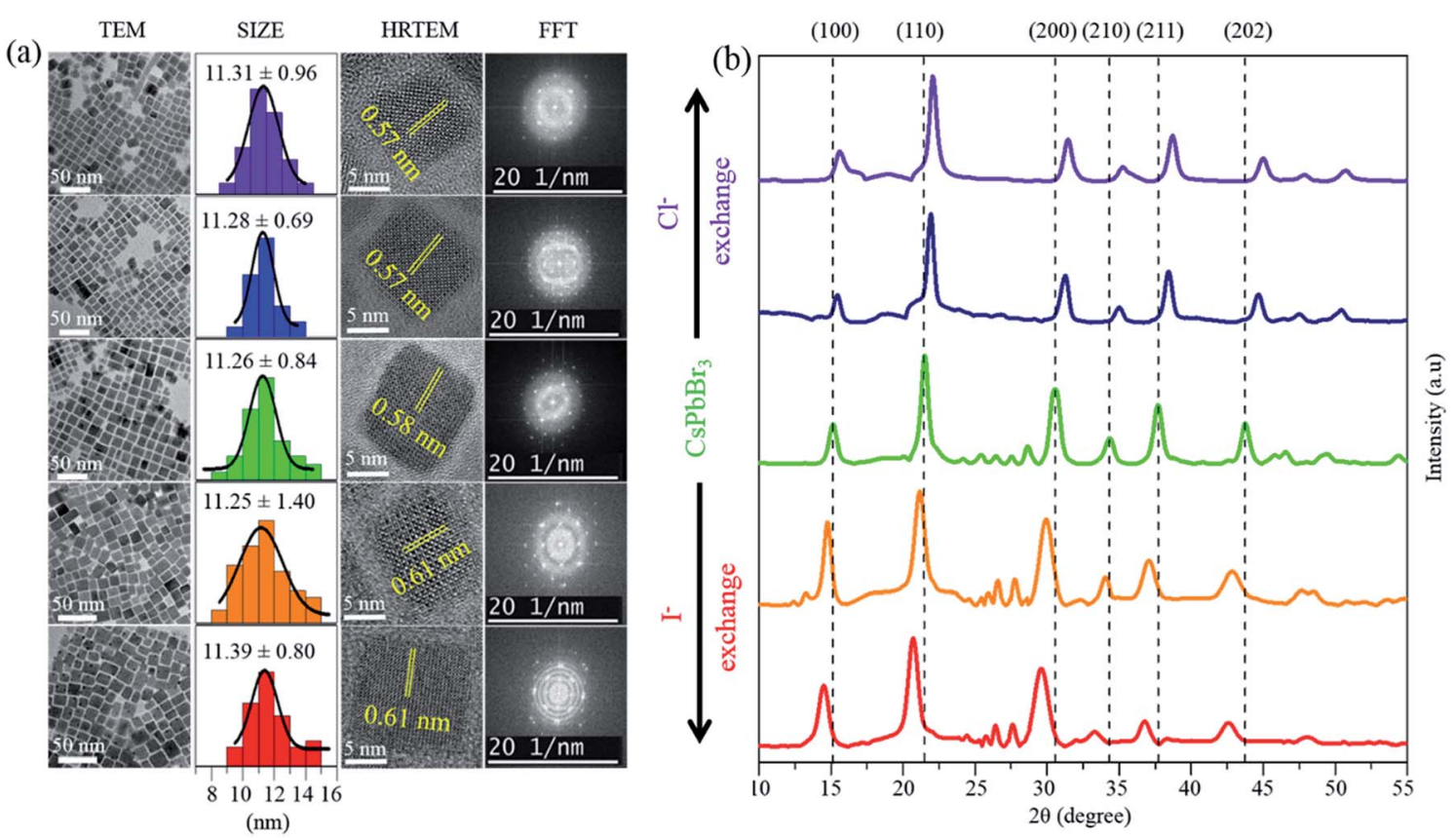

Fig. 1 Structural characterization of PQDs. (a) TEM image analysis of PQDs. (b) XRD pattern of PQDs.

exchange of the larger sized $\mathrm{I}^{-}(2.20 \AA)$ in place of the smaller sized $\mathrm{Br}^{-}$, the inter-planar spacing increases from $0.58 \mathrm{~nm}$ to $0.61 \mathrm{~nm} .{ }^{18}$ The shifting of the peaks corresponding to the (100), (110), (200), (210), (211), and (202) planes in the PXRD pattern (Fig. 1(b)) confirms the successful halide exchange of $\mathrm{CsPBr}_{3}$ by both $\mathrm{Cl}^{-}$and $\mathrm{I}^{-}$. The shifting of the PXRD peak positions and changes in the inter-planar spacing in the HRTEM images are expected because of the size difference of halides. ${ }^{18,27}$ However, this should not have a very significant effect on the overall size of the PQD, and the same has been observed herein, i.e., the overall sizes of all PQDs are in the range from 11.26 to $11.39 \mathrm{~nm}$. It has been reported in the literature that if the peaks corresponding to the $2 \theta$ values of $\sim 15^{\circ}$ and $32^{\circ}$ do not exhibit any significant splitting, the $\mathrm{PQD}$ crystal is cubic in nature. ${ }^{22}$ As can be observed from Fig. 1(b), in our case, the peaks corresponding to the $2 \theta$ values of $\sim 15^{\circ}$ and $32^{\circ}$ do not exhibit any significant splitting. Hence, based on the literature studies, we would like to conclude that our PQDs are cubic in nature. ${ }^{14,18,22,27}$ Moreover, note that none of the peaks in the PXRD pattern shows splitting in our case. The single peak positions without splitting confirm the uniform homogeneous nature of the PQDs formed because of halide exchange. ${ }^{22}$

Thus, we can conclude that the IRTOA method of the solution-phase halide exchange reaction does not alter the inner cationic sub-lattice. ${ }^{22}$ Preservation of the size and shape and the cubic nature of the high-quality PQD crystals are indicative of the ingenuity of the halide exchange process adopted in our study.

Moreover, we performed the IRTOA synthesis of several PQDs using $\mathrm{HCl}$ and $\mathrm{HI}$ as halide sources. Herein, note that the instantaneous halide exchange reactions were nearly quantitative as we never observed any PL peak corresponding to themother PQD $\mathrm{CsPbBr}_{3}$. As can be seen from Fig. 2(a) with a series of these pure and mixed halide all-inorganic PQDs we could cover emission wavelength ranged from blue $(430 \mathrm{~nm})$ to red (650 nm) (Fig. 2(b)).

The PL emission spectra are quite narrow (FWHM varying from 17 to $39 \mathrm{~nm}$ ) (Fig. 2(c)). As the size/atomic number of the halide increases, the FWHM of the PL emission band also increases. ${ }^{27}$ Quite interestingly, the mixed halide Br/I PQDs exhibit PL emission spectra that are narrower (FWHM $\sim 35 \mathrm{~nm}$ )
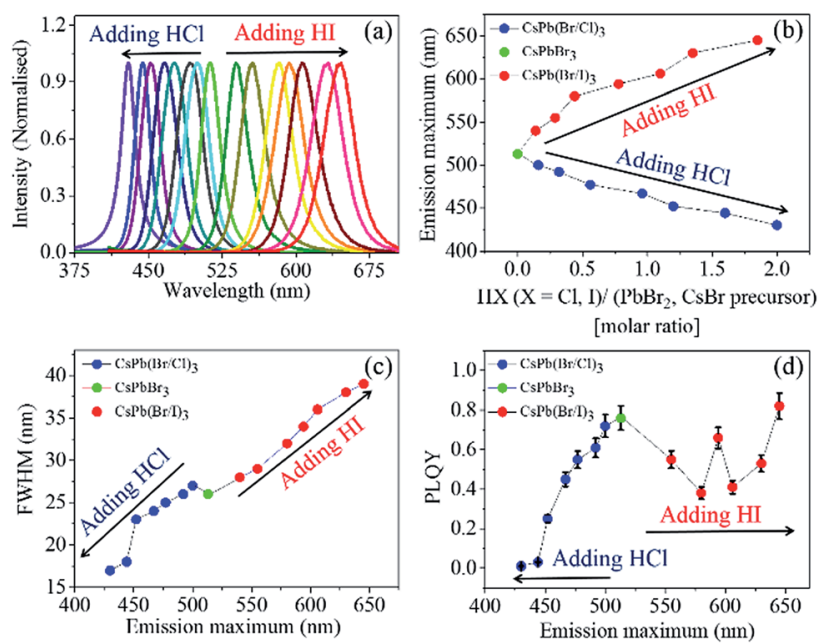

Fig. 2 (a) PL emission spectra of the PQDs prepared by halide exchange from $\mathrm{CsPbBr}_{3}$. (b) Evolution of the emission maximum with the addition of $\mathrm{HCl}$ and $\mathrm{HI}$ to a fixed amount of the $\mathrm{CsPbBr}_{3}$ solution. (c) Evolution of FWHM with halide exchange. (d) Evolution of PLQY with halide exchange. 
than those reported in the literature $(\mathrm{FWHM} \sim 50 \mathrm{~nm})^{\mathbf{1 8 , 1 9 , 2 7}}$ for the PQDs synthesized at high temperatures.

In literature, it is generally shown that the PLQY of $\mathrm{CsPbBr}_{3}$ is highest, and it decreases with the formation of the mixed halide PQDs $\mathrm{CsPb}(\mathrm{Br} / \mathrm{Cl})_{3}$ and $\mathrm{CsPb}(\mathrm{Br} / \mathrm{I})_{3} \cdot{ }^{19}$ The PLQY values reported herein for the $\mathrm{CsPb}(\mathrm{Br} / \mathrm{Cl})_{3}$ and $\mathrm{CsPb}(\mathrm{Br} / \mathrm{I})_{3}$ PQDs synthesized through the IRTOA process are either similar to or the same as those reported for the PQDs synthesized following several hours syntheses under an inert atmosphere through the hot injection procedure. ${ }^{18,19,27,29}$ In the case of $\mathrm{CsPb}(\mathrm{Br} / \mathrm{Cl})_{3}$, the magnitude of PLQY decreases monotonically as the relative percentage of chlorine increases. However, in the case of $\mathrm{CsPb}(\mathrm{Br} / \mathrm{I})_{3}$, the PLQY value initially decreases with a decrease in the concentration of $\mathrm{CsPbBr}_{3}$, whereas it increases beyond the PL maximum of $600 \mathrm{~nm}$; this may perhaps be because of the lowering of the conduction band energy level with respect to the low-lying trap states with an increase in the relative percentage of iodine. ${ }^{30}$

For example, for $\mathrm{CsPb}(\mathrm{Br} / \mathrm{I})_{3}$ PQDs (synthesized through the hot-injection inert atmosphere method), the PLQY values reported in most of the studies are $\sim 70 \% .{ }^{27}$ Herein, we have reported a similar PLQY value for the Br/I mixed halide PQDs (a maximum of $82 \%$ ) (Fig. 2(d)). The PLQY values of our $\mathrm{CsPb}(\mathrm{Br} /$ $\mathrm{Cl})_{3}$ PQDs are lower than that of $\mathrm{CsPbBr}_{3}$, and a similar trend has been observed for the $\mathrm{CsPb}(\mathrm{Br} / \mathrm{Cl})_{3}$ PQDs synthesized through the inert-atmosphere, hot-injection technique. ${ }^{19}$ Thus, the optical quality of the PQDs synthesized through the IRTOA method employing non-metal-based halide sources, such as $\mathrm{HCl} / \mathrm{HI}$, is similar to that of the PQDs synthesized through the high-temperature, inert-atmosphere synthesis for several hours. As a next step, we probed the PL behaviour of these PQDs using pico-nano second-based time-correlated single photon counting (TCSPC) and femtosecond-based transient absorption techniques. As can be observed from Fig. 3(a) (TCSPCbased PL decay monitored at PL emission maximum), as the halide changes from $\mathrm{Cl}$ to $\mathrm{Br}$ to I, the PL decay becomes slower in nature. In other words, as the size/atomic number of the halide increases, the PL decay becomes much slower (Table $\mathrm{S} 1 \dagger)$. In literature, for the PQDs synthesized through the hotinjection, inert-atmosphere synthesis, a similar pattern of PL decay has been reported. ${ }^{\mathbf{1 4 2 7}}$ The higher excited-state lifetime and broader PL emission spectra for the I-exchanged PQDS possibly indicate that low lying triplet states are involved in the PL emission process for the I-exchanged PQDs. Very recently, similar theoretical results have been reported in the literature. ${ }^{31}$ Detailed investigations in this direction are currently underway. To investigate whether our PQDs exhibit similar ultrafast dynamics as that reported in the literature at the femtosecond time scale, we have explored $\mathrm{CsPbBr}_{3}$ as a case study material with the excitation wavelength of $400 \mathrm{~nm}$ as this particular wavelength has been used in most of the studies reported in the literature on PQDs. As can be observed from Fig. 3(b), the bleach dynamics of the $\mathrm{CsPbBr}_{3}$ PQD monitored at $501 \mathrm{~nm}$ yielded a rise followed by a decay phenomenon. The rising part of the plot could be fitted with the time constant of $590( \pm 20) \mathrm{fs}$, and the decay part of the dynamics could be fitted with $50( \pm 10)$ and $250( \pm 50) \mathrm{ps}$. These values are quite similar to those reported in
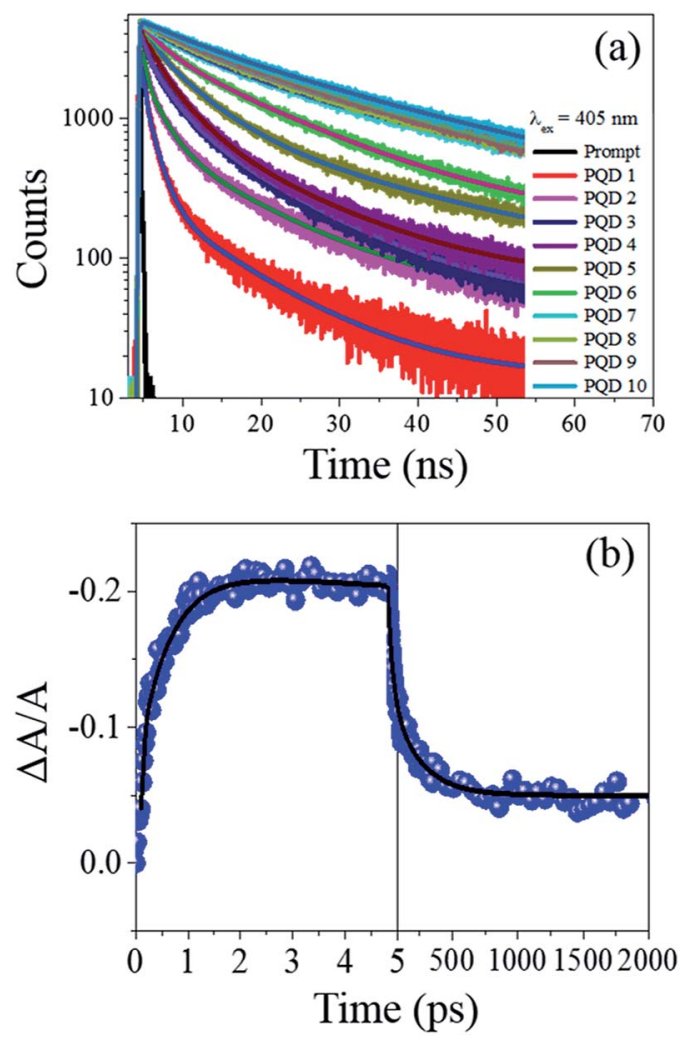

Fig. 3 Fast $\mathrm{PL}$ decay of different PQDs (a). Ultrafast (b) optical behaviour of $\mathrm{CsPbBr}_{3} \mathrm{PQD}$. Pump wavelength $=400 \mathrm{~nm}$.

the literature for PQDs. ${ }^{32-34}$ Thus, we can conclude that the PQDs synthesized herein by the IRTOA method employing halide exchange with inexpensive $\mathrm{HX}(\mathrm{X}=\mathrm{Cl} / \mathrm{I})$ exhibit similar ultrafast dynamics as that exhibited by the PQDs synthesized via the time-consuming, inert-atmosphere, hot-injection procedure. Detailed ultrafast dynamical analyses of other PQDs are currently under investigation.

As a next step, we probed how these PQDs would behave at the extremely sensitive single particle level. As the PLQY of these PQDs is quite high, these PQDs can be easily observed using our home-built total internal reflection (TIRF)-based single molecule spectroscopic setup (Fig. 4(a)). ${ }^{35}$ We carried out single particle measurements for the $\mathrm{PQD} 3(\mathrm{Br} / \mathrm{Cl}$ mixed halide $\left.\mathrm{PQD}, \lambda_{\mathrm{em}}=477 \mathrm{~nm}\right), \mathrm{PQD} 5\left(\mathrm{CsPbBr}_{3}, \lambda_{\mathrm{em}}=513 \mathrm{~nm}\right)$ and PQD 6 $\left(\mathrm{Br} / \mathrm{I}\right.$ mixed halide PQD, $\left.\lambda_{\mathrm{em}}=555 \mathrm{~nm}\right)$ with $405 \mathrm{~nm} \mathrm{CW}$ laser excitation and $100 \mathrm{~ms}$ binning time. Note that to date, the single particle spectroscopic results for three different PQDs $\left((\mathrm{Br} / \mathrm{Cl})_{3}\right.$, $\mathrm{Br}_{3}$, and $\left.(\mathrm{Br} / \mathrm{I})_{3}\right)$ under the same experimental conditions have not been reported in the literature. Typical single particle time traces are shown in Fig. 4(b). Blinking of these PQD single particles can be visualized clearly (Movie S7 $\dagger$ ). As observed from Fig. 4(c), for the PQD 3, i.e., PQD with the mixed $\mathrm{Br} / \mathrm{Cl}$ halide, a well separated bimodal distribution of the PL intensities was obtained, and the threshold was maintained at $3 \sigma$ above the peak intensity of the lower intensity distribution (where $\sigma$ is the standard deviation of the lower intensity distribution). Thus, a clear blinking behaviour with distinct ON and OFF events 

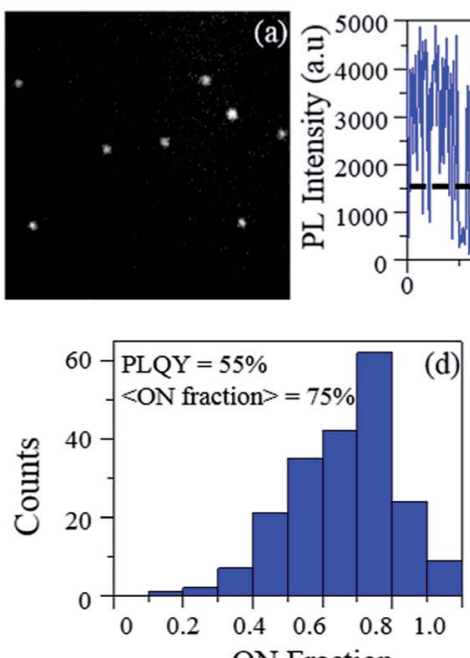

ON Fraction
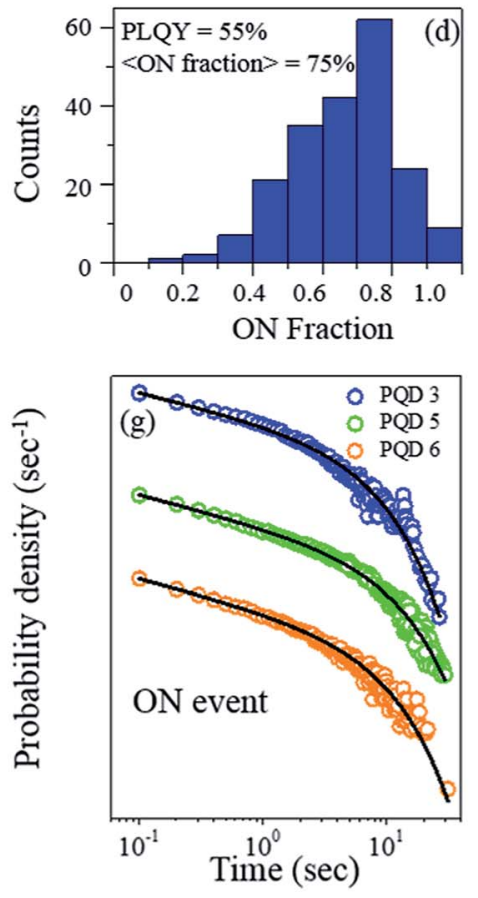

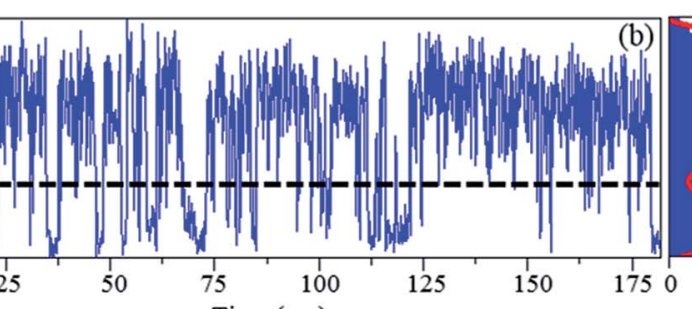

Time (sec)
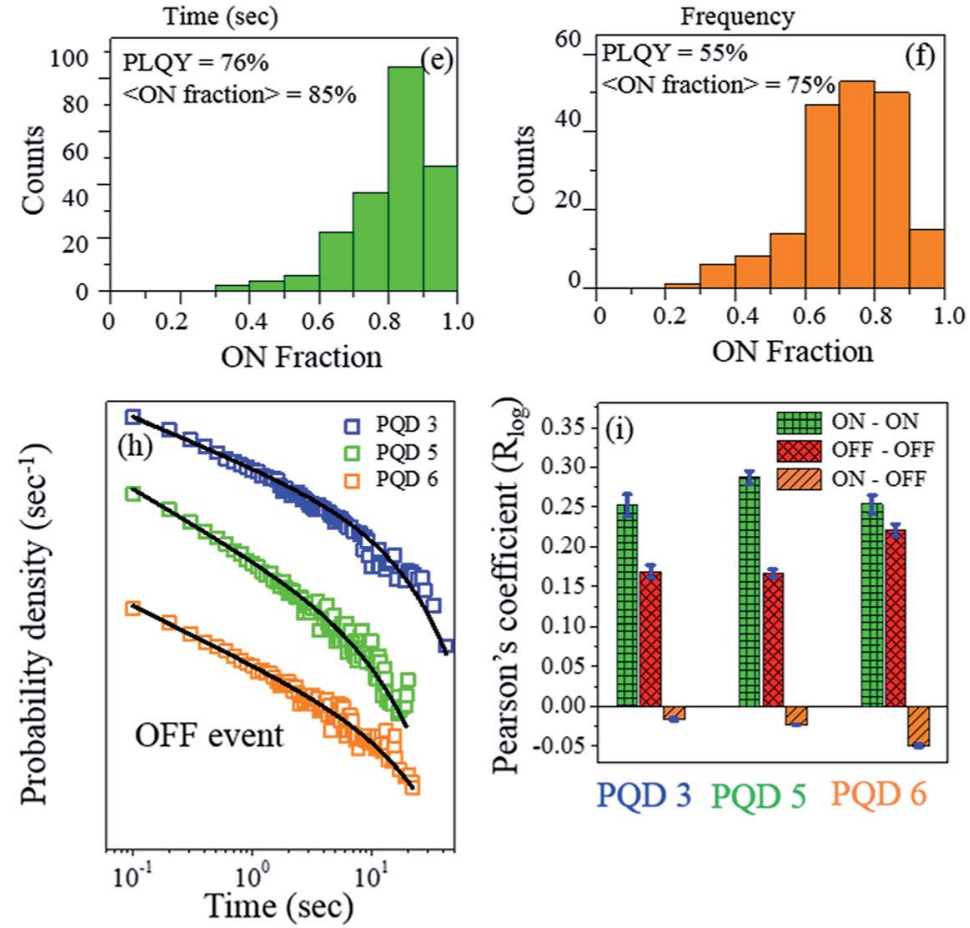

Fig. 4 (a) Single particle image with the PQD 3. (b) Single particle time trace with PQD 3. (c) PL intensity histogram. Percentage ON fraction for three PQDs: ((d) PQD 3, (e) PQD 5, and (f) PQD 6). Ensemble level PLQY values have been mentioned in the inset. Probability density distribution of the (g) ON event duration and (h) OFF event duration of all three PQDs. (i) Pearson's logarithm correlation coefficient ( $\left.R_{\text {log }}\right)$ of subsequent event durations (ON-ON, OFF-OFF, and ON-OFF) for all three PQDs.

could be noticed (for other PQDs, see Fig. S8†). The horizontal dashed line shown in Fig. 4(b) indicates the threshold value adopted herein; above this threshold value, the PL intensity is considered to be ON, and below this threshold value, the PL intensity is considered to be OFF.

From the PQD $3\left(\lambda_{\mathrm{em}}=477 \mathrm{~nm}\right)$ to PQD $5\left(\lambda_{\mathrm{em}}=513 \mathrm{~nm}\right)$ to PQD $6\left(\lambda_{\mathrm{em}}=555 \mathrm{~nm}\right)$, the percentage of the ON fraction initially increases from $75 \%$ to $85 \%$ and then decreases to $75 \%$ (Fig. 4(d), (e) and to Fig. 4(f), respectively). The ensemble level PLQY values for the respective PQDs have been mentioned in the inset of Fig. 4(d)-(f). The single particle level average \% ON fraction values of the respective PQDs seem to be correlated with the ensemble level PLQY values. The percentage of PQDs having the $\mathrm{ON}$ fraction higher than $70 \%$ is $\sim 50$ for the PQD 3, $\sim 85$ for the PQD 5, and $\sim 60$ for the PQD 6. Hence, at the single particle level, a large \% of the PQD population has high ON fraction value even after halide exchange with both $\mathrm{Cl}^{-}$and $\mathrm{I}^{-}$. Herein, note that the higher the ON fraction, the higher the probability of visualization of PQDs for the single particle tracking; moreover, the higher the percentage ON fraction, the better the quality of the PQD. Thus, we can conclude that at the single particle level, the $\mathrm{CsPBr}_{3} \mathrm{PQD}$ and the mixed halide PQDs (PQD 3 and PQD 6) remain mostly in the ON-state. These high ON fraction values are mostly similar to the values obtained for the PQDs synthesized through the inert atmosphere, hot-injection synthesis. ${ }^{36-38}$ This, in turn, demonstrates the ingenuity of the halide exchange process adopted herein. Thus, the IRTOA halide exchange reaction with inexpensive HX (X = $\mathrm{Cl} / \mathrm{I}$ ) yields PQDs with equally good (if not better) optical properties.

To investigate the blinking dynamics in detail, we calculated the probability density of the $\mathrm{ON}$ and $\mathrm{OFF}$ event durations (Fig. 4(g) (ON event durations) and Fig. 4(h) (OFF event durations)). For all three PQDs, the probability density distributions of both the ON and the OFF event durations truncate from the power law behaviour at longer event durations and are best fitted with a truncated power law equation (Fig. S9†). ${ }^{38,39}$ The power law exponent $(m)$ is $<1.0$ for the ON events $\left(m_{\mathrm{ON}}\right)$ and $>1.50$ for the OFF events ( $m_{\mathrm{OFF}}$ ) for all three PQDs (i.e., for all the samples, the $m_{\mathrm{OFF}}$ magnitude is higher than $m_{\mathrm{ON}}$ (Table 1)). As can be observed from Table 1, the ' $m$ ' value for a particular event (ON or OFF) does not change significantly from PQD 3 to PQD 5 
Table 1 Correlation between ensemble and single particle level optical properties of different PQDs

\begin{tabular}{lllllll}
\hline & & & & & & OFF \\
\cline { 3 - 4 } PQD & PLQY & $m_{\text {ON }}$ & ON truncation time (s) & & $m_{\text {OFF }}$ & OFF truncation time (s) \\
\hline PQD 3 & $0.55 \pm 0.02$ & $0.90 \pm 0.04$ & $2.7 \pm 0.1$ & $1.55 \pm 0.06$ & $5.6 \pm 0.2$ \\
PQD 5 & $0.76 \pm 0.02$ & $0.94 \pm 0.05$ & $4.0 \pm 0.2$ & $1.85 \pm 0.10$ & $4.3 \pm 0.2$ \\
PQD 6 & $0.55 \pm 0.03$ & $0.95 \pm 0.05$ & $2.9 \pm 0.1$ & & $1.80 \pm 0.09$ & $6.6 \pm 0.3$ \\
\hline
\end{tabular}

to PQD 6. However, for a particular sample, the difference between the ' $m$ ' values for the $\mathrm{ON}$ and OFF events is quite significant (Table 1). For a particular sample, the relative magnitude of ' $m$ ' will dictate which event (ON or OFF) will be more probable for a particular event duration. Hence, the ' $m$ ' values become important while comparing the probability density distribution of the ON and OFF events for a particular sample. A relatively higher value of $m_{\mathrm{OFF}}$ than that of $m_{\mathrm{ON}}$ suggests (1.5 as compared to 0.9 ) that the slope of the ON event probability density distribution is less steeper than that of the OFF event probability density distribution. This, in turn, signifies that the probability of the ON event durations will be higher than that of the OFF event durations. ${ }^{\mathbf{4 0 , 4 1}}$ After halide exchanges with the $\mathrm{Cl}^{-}$and $\mathrm{I}^{-}$ions, the PLQY decreases from 76\% (PQD 5) to 55\% for both PQD 3 and PQD 6 (Table 1). A similar trend was also observed for the $\mathrm{ON}$ event truncation time, which decreased from $4.0 \mathrm{~s}$ (PQD 5) to $2.7 \mathrm{~s}$ for the $\mathrm{Cl}^{-}$ exchange (PQD 3) and to $2.9 \mathrm{~s}$ for the $\mathrm{I}^{-}$exchange (PQD 6) (Table 1). However, an opposite trend was observed for the OFF event truncation times, which increased from $4.3 \mathrm{~s}$ (PQD 5) to $5.6 \mathrm{~s}$ (PQD 3) and 6.6 s (PQD 6) (Table 1). A decrease in the ON event truncation time (from PQD 5 to PQD 3/PQD 6) suggests that the ON event distributions truncate earlier (in PQD 3/PQD 6 as compared to those in PQD 5); this would make the longer ON events less probable after halide exchange.

Similarly, the longer OFF events become more probable (in PQD 3/PQD 6 as compared to those in PQD 5) as the OFF event duration distribution truncates later in the mixed halide PQDs (PQD 3/PQD 6). Thus, for different PQD samples, an increase in the $\mathrm{ON}$ event truncation time and concomitant decrease in the OFF event truncation time could be correlated with the increase in the PLQY values. Thus, we could successfully correlate the physical parameters obtained from the ensemble as well as the single particle level.

We also studied the presence of the memory effect in all three PQD samples. Although studies have been reported on the memory effects in QDs, ${ }^{\mathbf{4 1 - 4 4}}$ the same has not been explored for the all-inorganic PQDs to date. The two-dimensional (2D) joint probability distribution of the adjacent event durations (logarithms of event duration) for both ON and OFF events has been plotted with colour coding (Fig. S11 $\dagger$ ). A 2D joint probability distribution of the adjacent ON events (ON event to next ON event) for all three PQDs has been plotted in Fig. S11(a, g, and $\mathrm{m}), \dagger$ and Fig. $\mathrm{S} 11(\mathrm{~b}, \mathrm{~h}$, and $\mathrm{n}) \dagger$ represent the same for the $\mathrm{ON}$ events that are sufficiently separated (e.g., $1^{\text {st }}$ ON event to $50^{\text {th }}$ ON event). Then, the latter has been subtracted from the former. The subtracted 2D joint probability distribution (Fig. S11(c, i, and o) $\dagger$ ) in the matrix plot shows a significant diagonalization, indicating the presence of residual memory in the ON event durations. ${ }^{\mathbf{4 1 , 4 2}}$ Similar analyses on the OFF event durations also show the presence of memory among the OFF event durations (Fig. S11(f, l, and r) $\dagger$ ).

To quantify the residual memory, Pearson's logarithm correlation coefficient for the adjacent event durations was calculated. For all three PQDs, both ON-ON and OFF-OFF events are positively correlated; however, the ON-OFF events are negatively correlated (Fig. 4(i)). The magnitude of the Pearson's logarithm correlation coefficient for the ON-ON events is higher than that for the OFF-OFF events. Similar results have been reported for the CdSe and InP-based QDs. ${ }^{41-46}$ As can be observed from Fig. 4(i), the ON-OFF events are anti-correlated. Thus, the existence of a residual memory in the all-inorganic pure $\mathrm{CsPbBr}_{3}$ and mixed halide PQDs could be evidenced.

We have compared our result with those of two of the recently reported studies. ${ }^{21,47}$ First, in these two studies, the authors have synthesized $\mathrm{CsPbBr}_{3}$ via the hot-injection method under an inert atmosphere condition. This is different from the way we have synthesized $\mathrm{CsPbBr}_{3}$, i.e., via the IRTOA method. Second, in both the studies, ${ }^{\mathbf{2 1}, \mathbf{4 7}}$ the chloride/bromide exchange has been achieved using $\mathrm{HCl} / \mathrm{HBr}$ in the vapour phase. Moreover, in one study, ${ }^{21}$ the $\mathrm{Br} \rightarrow \mathrm{I}$ anion exchange has not been performed by HI. Instead, the authors have used an OLAM-I precursor, which has been synthesized by the reaction of oleylamine (OAM) with $\mathrm{I}_{2}$ overnight.

In the other study, ${ }^{46}$ the I exchange has been achieved by $\mathrm{HI}$ vapour towards a sensing purpose. In our process, all halide exchanges were performed using $\mathrm{HX}(\mathrm{X}=\mathrm{Cl} / \mathrm{Br} / \mathrm{I})$ through the IRTOA method in the solution phase and not in the vapour phase for sensing purpose as abovementioned in this study. Third, neither of the reported ${ }^{\mathbf{2 1 , 4 7}}$ vapour phase halide replacements was instantaneous, and it took 20-60 minutes for the halide exchange. However, in our case, all halide exchanges took place instantaneously. Fourth, in none of these reports, ${ }^{21,47}$ quantitative procedures of mixed halide PQD synthesis covering a large range of PL emission maxima have been reported. However, in our case, we have not only synthesized mixed halide PQD with a range of PL emission maxima, but also characterized them all using TEM, EDAX, PXRD, among other techniques. Thus, in these two literature studies, ${ }^{21,47}$ (i) $\mathrm{CsPbBr}_{3}$ has been synthesized via the hot-injection inert atmosphere method, and then, (ii) vapour phase halide exchange was conducted, (iii) which took 20-60 minutes, and (iv) by this 
procedure, only one mixed halide PQD with a fixed PL emission maximum can be synthesized. However, in this study, we report (i) the synthesis of $\mathrm{CsPbBr}_{3}$ via the RT open-air atmosphere method. Then, the mixed halide PQDs were synthesized in (ii) the solution phase, (iii) instantaneously, and (iv) a wide range of PL emission maxima (from $430 \mathrm{~nm}$ to $650 \mathrm{~nm}$ ) could be covered. Thus, our study is significantly different from the abovementioned two literature studies.

\section{Conclusions}

In conclusion, herein, the instantaneous synthesis of blue to green to yellow to red-emitting all-inorganic perovskite quantum dots (PQDs) was achieved at room temperature under an open-air atmosphere (no glove box) through halide exchange from $\mathrm{CsPbBr}_{3}$ employing non-metal based inexpensive and easily available halide sources such as $\mathrm{HCl}$ and HI. All PQDs were highly crystalline and cubic-shaped with the size uniformity of $c a .11 .3 \pm 0.1 \mathrm{~nm}$. These PQDs were stable for a few weeks under an open-air atmosphere. The PL emission spectra are quite narrow, and the PLQY values are quite high (80\% for even Br/I mixed PQDs). A very regular increase in the PL lifetime has been observed as the halide changes from $\mathrm{Cl}$ to $\mathrm{Br}$ to $\mathrm{I}$. Ultrafast dynamics revealed the excited-state time constants at the femtosecond to nanosecond timescales, similar to those of PQDs synthesized through the hot injection, inert atmosphere procedure. This kind of room-temperature (no use of heat energy/electrical energy), open-air atmosphere (no use of cumbersome inert atmosphere), instantaneous (less time consuming), nearly quantitative synthesis of PQDs using inexpensive and easily available $\mathrm{HCl} / \mathrm{HI}$ is extremely important for the large-scale synthesis of PQDs for various applications. At the single particle level, the $\langle\mathrm{ON}$ fraction $\rangle$ has been noted to vary from $75 \%$ to $85 \%$ for different PQDs; this indicates that the blue-green-yellow-red-emitting PQDs are quite bright and mostly in the ON state even at the single particle level. The magnitudes of the $m_{\mathrm{ON}}$ values are close to 1.0 , and the $m_{\mathrm{OFF}}$ values are $>1.5$, indicating that long ON durations are more probable. The increase in the ON event truncation time (from 2.7 to $4.0 \mathrm{~s}$ ) and a concomitant decrease in the OFF event truncation time (from 6.6 to $4.3 \mathrm{~s}$ ) could be correlated with an increase in the PLQY (from 0.55 to 0.75 ). An interesting memory effect could be observed in both the ON and the OFF event durations.

\section{Conflicts of interest}

There are no conflicts to declare.

\section{Acknowledgements}

P. K. M. thanks IISER Kolkata for financial help and instrumental facilities. Support received from the CSIR India, Project No. 01(2848)/16/EMR-II is gratefully acknowledged. S. M. thanks IISER Kolkata; D. R., M. M., and A. D. thank CSIR; C. K. D. and S. G. thank DST-INSPIRE for the fellowship. The authors thank Dr Prakriti Ranjan Bangal (IICT, India) for the femtosecond measurement.

\section{References}

1 J. Liang, C. Wang, Y. Wang, Z. Xu, Z. Lu, Y. Ma, H. Zhu, Y. Hu, C. Xiao, X. Yi, G. Zhu, H. Lv, L. Ma, T. Chen, Z. Tie, Z. Jin and J. Liu, J. Am. Chem. Soc., 2016, 138, 15829.

2 Q. A. Akkerman, M. Gandini, F. Di Stasio, P. Rastogi, F. Palazon, G. Bertoni, J. M. Ball, M. Prato, A. Petrozza and L. Manna, Nat. Energy, 2016, 2, 16194.

3 S. Christodoulou, F. Di Stasio, S. Pradhan, A. Stavrinadis and G. Konstantatos, J. Phys. Chem. C, 2018, 122, 7621.

4 S. Yakunin, L. Protesescu, F. Krieg, M. I. Bodnarchuk, G. Nedelcu, M. Humer, G. De Luca, M. Fiebig, W. Heiss and M. V. Kovalenko, Nat. Commun., 2015, 6, 8056.

5 Y. Fu, H. Zhu, C. C. Stoumpos, Q. Ding, J. Wang, M. G. Kanatzidis, X. Zhu and S. Jin, ACS Nano, 2016, 10, 7963. 6 Y. Xu, Q. Chen, C. Zhang, R. Wang, H. Wu, X. Zhang, G. Xing, W. W. Yu, X. Wang, Y. Zhang and M. Xiao, J. Am. Chem. Soc., 2016, 138, 3761.

7 J. Song, J. Li, X. Li, L. Xu, Y. Dong and H. Zeng, Adv. Mater., 2015, 27, 7162.

8 Q. Shan, J. Li, J. Song, Y. Zou, L. Xu, J. Xue, Y. Dong, C. Huo, J. Chen, B. Han and H. Zeng, J. Mater. Chem. C, 2017, 5, 4565. 9 J. S. Yao, J. Ge, B. N. Han, K. H. Wang, H. B. Yao, H. L. Yu, J. H. Li, B. S. Zhu, J. Z. Song, C. Chen, Q. Zhang, H. B. Zeng, Y. Luo and S. H. Yu, J. Am. Chem. Soc., 2018, 140, 3626.

10 H. Zhang, X. Wang, Q. Liao, Z. Xu, H. Li, L. Zheng and H. Fu, Adv. Funct. Mater., 2017, 27, 1604382.

11 M. Kulbak, D. Cahen and G. Hodes, J. Phys. Chem. Lett., 2015, 6, 2452.

12 M. Kulbak, S. Gupta, N. Kedem, I. Levine, T. Bendikov, G. Hodes and D. Cahen, J. Phys. Chem. Lett., 2016, 7, 167.

13 J. Song, L. Xu, J. Li, J. Xue, Y. Dong, X. Li and H. Zeng, Adv. Mater., 2016, 28, 4861.

14 L. Protesescu, S. Yakunin, M. I. Bodnarchuk, F. Krieg, R. Caputo, C. H. Hendon, R. X. Yang, A. Walsh and M. V. Kovalenko, Nano Lett., 2015, 15, 3692.

15 Y. Bekenstein, B. A. Koscher, S. W. Eaton, P. Yang and A. P. Alivisatos, J. Am. Chem. Soc., 2015, 137, 16008.

16 D. Zhang, S. W. Eaton, Y. Yu, L. Dou and P. Yang, J. Am. Chem. Soc., 2015, 137, 9230.

17 J. Shamsi, Z. Dang, P. Bianchini, C. Canale, F. D. Stasio, R. Brescia, M. Prato and L. Manna, J. Am. Chem. Soc., 2016, 138, 7240.

18 G. Nedelcu, L. Protesescu, S. Yakunin, M. I. Bodnarchuk, M. J. Grotevent and M. V. Kovalenko, Nano Lett., 2015, 15, 5635.

19 Q. A. Akkerman, V. D'Innocenzo, S. Accornero, A. Scarpellini, A. Petrozza, M. Prato and L. Manna, J. Am. Chem. Soc., 2015, 137, 10276.

20 A. Haque, V. K. Ravi, G. S. Shanker, I. Sarkar, A. Nag and P. K. Santra, J. Phys. Chem. C, 2018, 122, 13399.

21 F. Palazon, Q. A. Akkerman, M. Prato and L. Manna, ACS Nano, 2016, 10, 1224. 
22 S. Fang, G. Li, Y. Lu and L. Li, Chem.-Eur. J., 2018, 24, 1898. 23 S. Sun, D. Yuan, Y. Xu, A. Wang and Z. Deng, ACS Nano, 2016, 10, 3648.

24 S. Seth and A. Samanta, Sci. Rep., 2016, 6, 37693.

25 C. Guhrenz, A. Benad, C. Ziegler, D. Haubold, N. Gaponik and A. Eychmüller, Chem. Mater., 2016, 28, 9033.

26 S. K. Balakrishnan and P. V. Kamat, ACS Energy Lett., 2016, 2, 88.

27 C. Bi, S. Wang, W. Wen, J. Yuan, G. Cao and J. Tian, J. Phys. Chem. C, 2018, 122, 5151.

28 X. Li, Y. Wu, S. Zhang, B. Cai, Y. Gu, J. Song and H. Zeng, Adv. Funct. Mater., 2016, 26, 2435.

29 Y. Su, X. Chen, W. Ji, Q. Zeng, Z. Ren, Z. Su and L. Liu, ACS Appl. Mater. Interfaces, 2017, 9, 33020.

30 A. Swarnkar, V. K. Ravi and A. Nag, ACS Energy Lett., 2017, 2, 1089.

31 M. A. Becker, R. Vaxenburg, G. Nedelcu, P. C. Sercel, A. Shabaev, M. J. Mehl, J. G. Michopoulos, S. G. Lambrakos, N. Bernstein, J. L. Lyons, T. Stoferle, R. F. Mahrt, M. V. Kovalenko, D. J. Norris, G. Raino and A. L. Efros, Nature, 2018, 553, 189.

32 P. Maity, J. Dana and H. N. Ghosh, J. Phys. Chem. C, 2016, $120,18348$.

33 N. Mondal and A. Samanta, Nanoscale, 2017, 9, 1878.

34 N. Soetan, A. Puretzky, K. Reid, A. Boulesbaa, H. F. Zarick, A. Hunt, O. Rose, S. Rosenthal, D. B. Geohegan and R. Bardhan, ACS Photonics, 2018, 5, 3575.
35 D. Roy, T. Routh, A. V. Asaithambi, S. Mandal and P. K. Mandal, J. Phys. Chem. C, 2016, 120, 3483-3491.

36 A. Swarnkar, R. Chulliyil, V. K. Ravi, M. Irfanullah, A. Chowdhury and A. Nag, Angew. Chem., Int. Ed., 2015, 54, 15424.

37 A. Zhang, C. Dong and J. Ren, J. Phys. Chem. C, 2017, 121, 13314.

38 N. A. Gibson, B. A. Koscher, A. P. Alivisatos and S. R. Leone, J. Phys. Chem. C, 2018, 122, 12106-12113.

39 G. Yuan, C. Ritchie, M. Ritter, S. Murphy, D. E. Gómez and P. Mulvaney, J. Phys. Chem. C, 2018, 122, 13407-13415.

40 A. A. Cordones and S. R. Leone, Chem. Soc. Rev., 2013, 42, 3209-3221.

41 D. Roy, S. Mandal, C. K. De, K. Kumar and P. K. Mandal, Phys. Chem. Chem. Phys., 2018, 20, 10332-10344.

42 F. D. Stefani, X. Zhong, W. Knoll, M. Han and M. Kreiter, New J. Phys., 2005, 7, 197.

43 J. P. Hoogenboom, J. Hernando, M. F. García-Parajó and N. F. van Hulst, J. Phys. Chem. C, 2008, 112, 3417-3422.

44 S. Volkán-Kacsó, P. A. Frantsuzov and B. Jankó, Nano Lett., 2010, 10, 2761-2765.

45 C. K. De, T. Routh, D. Roy, S. Mandal and P. K. Mandal, J. Phys. Chem. C, 2018, 122, 964-973.

46 D. Roy, A. Das, C. K. De, S. Mandal, P. R. Bangal and P. K. Mandal, J. Phys. Chem. C, 2019, 123, 6922-6933.

47 X. Chen, H. Hu, Z. Xia, W. Gao, W. Gou, Y. Qu and Y. Ma, J. Mater. Chem. C, 2017, 5, 309-313. 